\title{
How should we manage exsanguinating pelvic fractures in the United Kingdom?
}

\author{
Steve Meek, Raymond Ross
}

\begin{abstract}
Accident and
Emergency

Department, Bristol

Royal Infirmary,

Bristol, UK

S Meek

Orthopaedic Department, Hope

Hospital, Salford, UK E R S Ross

Correspondence to:

Ninety eight per cent of patients with major pelvic fractures have multiple injuries ${ }^{3}$ and intra-abdominal injury occurs in $18-35 \%^{3}$; thus the patient may be exsanguinating from the pelvis, from abdominal organs, or both, yet the diagnosis or exclusion of significant intraperitoneal haemorrhage is difficult and time is short. An unnecessary laparotomy will significantly reduce the chance of survival, but equally, overlooking a serious intra-abdominal injury may have fatal consequences.
\end{abstract} Mr S Meek, Consultant, Accident and Emergency Department, Royal United Hospital, Bath BA1 3NG, UK.

Accepted for publication 28 May 1997

\section{Where does the haemorrhage originate from?}

Exsanguinating pelvic haemorrhage usually originates from fractured bone ends and the presacral venous plexus, though some bleeding
Haemodynamically unstable patients with pelvic fractures present a difficult management problem in accident and emergency (A\&E) departments. There have been many reports supporting various treatment methods, though no prospective controlled trials of these different approaches have been undertaken. In this paper we shall review the evidence for the available treatment options and make recommendations for management of such patients in the United Kingdom.

Relevant publications were identified by a combination of techniques: a Medline search combining the terms "pelvic fracture" and "bleeding," then identifying relevant work from the abstract; scrutiny of the reference lists for all papers and examination of any likely to be relevant; personal communications leading to identification of two articles; and hand searching two journals with a high incidence of relevant papers. Only English language papers were used. Relevance to the topic was the only inclusion criterion. None of the papers reviewed was a controlled trial, though some involved experimental comparisons. Data were not combined since inclusion criteria were different in the different series.

The combination of pelvic ring fractures and hypotension following blunt trauma requires skilled and urgent management. A mortality rate of $42 \%$ has been reported for those patients with pelvic ring fractures who arrive haemodynamically unstable, compared with $3.4 \%$ for those arriving haemodynamically stable, ${ }^{1}$ and nearly $40 \%$ of early deaths from pelvic fractures are due to uncontrollable haemorrhage. ${ }^{2}$ may be arterial. Necropsy studies ${ }^{4}$ have indicated that bleeding is from cancellous bone ends in the posterior pelvic ring, supplied by posterior branches of the hypogastric (internal iliac) artery, suggesting that fracture realignment is likely to be the most effective strategy; this is supported by Latenser et al. ${ }^{5}$

European clinicians such as Ganz et $a l,{ }^{6}$ Pohlemann et $a{ }^{7}{ }^{7}$ Schutz et $a l^{8}{ }^{8}$ and Tscherne and Regel $^{9}$ are convinced by their personal experience that severe bleeding is almost always venous, usually from the presacral plexus. Many American clinicians feel that in a significant minority of cases there is a large arterial component. For example, BenManachem et $a l^{10}$ and other pro-angiography investigators believe that up to $14 \%$ of haemodynamically unstable pelvic ring fractures have arterial bleeding on angiography. Others such as Gylling $e t a l^{11}$ and Riemer $e t a l^{12}$ claim a low success rate with angiography and point to the failure of internal iliac artery ligation to control haemorrhage as clear evidence that severe bleeding is usually venous.

Whatever its origin, the magnitude of bleeding is much greater, and may go unrecognised, when disruption of the sacroiliac joints or posterior structures is present. McMurtry et $a l^{13}$ found that patients with such posterior instability required a mean of 15.5 units of blood, compared with 5.1 units in patients without posterior instability. Assuming the pelvic ring to be a sphere, the contained volume increase will be proportional to the fourth power of the radius. Latenser $e t a l^{14}$ calculated that a $3 \mathrm{~cm}$ diastasis of the pubic symphysis would thus double the available pelvic volume for haemorrhage before tamponade occurs, though this has recently been challenged by Moss and Bircher $^{15}$ : their model showed that even a 10 $\mathrm{cm}$ diastasis of the pubic symphysis, plus a 3 $\mathrm{cm}$ widening of a sacroiliac joint, will only increase true pelvic volume by $55 \%$. In any case, the haemorrhage can be accommodated by tracking up the retroperitoneal space, where a collection of up to 4 litres has been described. ${ }^{14}$

\section{Management options}

External fixation may be anterior or posterior. Anterior external fixation involves using a simple anterior metal frame fixed to the ilium with pins. This can be applied in the resuscitation 
room or operating theatre in 20 to 30 minutes by a competently trained doctor of any grade or background, and aims to tamponade the bleeding by reducing the pelvic volume and opposing fractured bone ends. Posterior external fixation is achieved by direct compression of the sacroiliac joints with a "C" clamp. ${ }^{6}$

Internal fixation is the strongest way to stabilise fractures in the pelvis. This has been used in conjunction with direct surgical control and packing by some European surgeons to control haemorrhage if external fixation is ineffective or as the primary method of haemorrhage control.

The pneumatic antishock garment is rarely used in the United Kingdom but seeks to achieve a reduction in pelvic volume and tamponade of abdomen, pelvis, and lower limbs by the inflation of various compartments of the suit with compressed air.

Angiography and embolisation is favoured by some American surgeons, but is infrequently used in Europe. This involves the identification of bleeding sites by a percutaneous approach and injection of Gelfoam or other material into the relevant artery to occlude it proximal to the bleeding - a highly skilled and time consuming procedure.

\section{EXTERNAL FIXATION}

All the published work on external fixation is either retrospective or was carried out on cadavers, and this should be borne in mind in assessing the evidence below.

The application of external fixation is recommended by most clinicians, though to varying degrees. An exception is Gruen et al, ${ }^{16}$ who prefer "early" open reduction and internal fixation, never using external fixation, while others such as Mucha and Welch ${ }^{17}$ use it acutely only in open book type fractures.

There are several publications claiming that overall mortality in bleeding pelvic fractures may be improved by the use of external fixation. Gylling et $a l^{11}$ reported results from three years' experience using "immediate" external fixation on all major unstable pelvic fractures; the mortality in these 26 patients was $12 \%$, the same as in 40 with stable fractures treated without any fixation. As this figure is much lower than in other series, ${ }^{17-19}$ the authors conclude external fixation is likely to decrease mortality. However, "immediate" meant within 24 hours of admission, and the paper gave no evidence of a reduction in haemorrhage, for example by a decrease in transfusion requirements after fixation.

Moreno et $a l^{2}$ claim just such a decrease in their retrospective review. Nineteen patients with a variety of pelvic fractures, all requiring more than six units of blood in the first 24 hours, were treated with external fixation for haemorrhage control: "bleeding was controlled" in $18 / 19$ (95\%), with a drop in mean transfusion requirements from 7.4 units to 3.7 after fixation. Unfortunately, very little detail is offered on this aspect of the paper, and the accuracy and timing of data obtained from retrospective review must be treated with caution.

Riemer $e t \mathrm{al}^{12}$ found a decrease in mortality in patients with pelvic fractures after the intro- duction of a protocol for early mobilisation and external fixation; however, the timing of the external fixation was variable and it was often performed to aid mobilisation rather than to reduce blood loss. Again this is a retrospective review and many other factors could have contributed to the fall in mortality.

External fixation may not be appropriate for all pelvic fractures. There is consensus that it is the treatment of choice in "open book" fractures, where an anterior frame will "close the book" effectively. Multidirectional fracture displacement will not be as well controlled by an anterior frame, and Mucha and Welch ${ }^{17}$ believe that using anterior frames in such patients will "do more harm than good." Tile, ${ }^{20}$ however, believes a reduction in bleeding is still achieved, perhaps by reducing pelvic volume and tamponade.

Using an anterior frame in lateral compression type fractures could be detrimental by increasing medial rotation and displacement of the fragments. ${ }^{2621}$

Mucha and Welch $^{17}$ state that anterior frames will be detrimental where there is an acetabular component, or a shattered ileum (where inserting the pins would be very difficult if not impossible), and these injury patterns seem to be logical contraindications to external fixation unless the frame is fixed to the femur on that side.

There is an inherent mechanical flaw in the use of anterior frames to improve pelvic fracture compression/motion in that they can only compress the anterior pelvis, relying on intact sacroiliac ligaments and bone posteriorly. If these are not intact, movements continue and volume increase can still occur, and it is for this reason that Ganz and his colleagues designed a posterior clamp (the "antishock" clamp) and reported haemodynamic improvement in two of five patients. ${ }^{6} \mathrm{He}$ recently reported results on a further 30 patients in a paper with Heini et al. ${ }^{22}$ Eighteen patients were haemodynamically unstable and they claim the frame (now called the AO C-clamp) was "haemodynamically effective" in 10 patients. Use of the Ganz clamp is also supported by Pohlemann et al, ${ }^{7}$ though the figures in this 21 year review suggest he and his colleagues in Hannover have used it in only 20 patients, and the effect on haemorrhage is not mentioned, perhaps as they favour direct surgical control of bleeding. Further German support for posterior fixation comes from Schutz et al, ${ }^{8}$ who compared the Ganz/AO C-clamp with the more recently developed ACE C-clamp. It was, they claimed, easier to achieve accurate pin alignment with the Ganz clamp, though with only five patients receiving the Ganz clamp and four the ACE clamp, no further conclusions can be drawn.

In contrast to the German/Swiss enthusiasm for posterior external fixation, the American investigators Wilson et $a l^{23}$ found the Ganz clamp ineffective at resisting intrapelvic pressure increases. They tested the Ganz clamp, various anterior frames, and methods of internal fixation on cadavers with unstable fractures using an intrapelvic balloon: the best 
resistance to intrapelvic pressure increase was from combined anterior and posterior internal fixation. All forms of anterior frame external fixation performed similarly, but they found the Ganz clamp provided negligible resistance to expansion.

There is no evidence to suggest any particular anterior frame is superior to others in effectiveness, though some are easier to apply than others. ${ }^{20} 23$

Laparotomy in a patient with haemorrhage from pelvic fractures can lead to a loss of tamponade and acceleration of bleeding. The principle that external fixation should be applied before the abdomen is opened is now widely accepted. Ghanayem et al $^{24}$ studied this by creating unstable pelvic fractures in cadavers. They found pelvic volume to be $26 \%$ larger in those not externally fixed before laparotomy. Therefore if an emergency laparotomy is indicated, unless surgical control of pelvic bleeding is to be attempted, external fixation should be applied before the skin incision.

INTERNAL FIXATION AND SURGICAL CONTROL OF BLEEDING

There is a marked difference between the Americans and the continental Europeans on this subject, with the Europeans such as Pohlemann, Ganz, Schutz, and Heini ${ }^{6-8} 22$ favouring direct haemorrhage control if possible, packing, and internal fixation, while the majority of American clinicians feel the surgery is difficult and ineffective, preferring to use angiographic embolisation. Mucha and Farnell $^{1}$ describe such surgery as "fraught with failure." Tile ${ }^{20}$ believes that identification of bleeding vessels and ligation is difficult and likely to be ineffective because of the rich collateral network in the pelvis, with a loss of tamponade when the peritoneum is incised. They claim that internal fixation is contraindicated in the first few hours for this reason, and favour embolisation.

In contrast, Goldstein et $a l^{25}$ favour early internal fixation, but to improve early mobilisation rather than for haemorrhage control, and use angiography and embolisation when necessary before surgery.

Gruen et $a l^{16}$ similarly favour early open reduction and internal fixation (ORIF) following angiographic embolisation to control bleeding, never using external fixation. In a retrospective study on 36 patients with pelvic ring fractures who were admitted in shock, three were successfully embolised before surgery, but two were still actively bleeding and underwent limited internal fixation for haemorrhage control, both surviving. Interestingly, both had open book fractures, which the vast majority of clinicians feel are a clear indication for external fixation, and their definition of early internal fixation was within 96 hours!

In contrast, the German and Swiss workers mentioned above have all developed aggressive management protocols involving early internal fixation and surgical control of bleeding. Usually this means packing the pelvis for 48 hours in all cases where continuing bleeding occurs after application of external fixation. Angiography is reserved for patients who are still bleed- ing after surgery. With such a protocol, Pohlemann et $\mathrm{al}^{7}$ retrospectively calculate a fall in mortality for complex pelvic fractures (those with soft tissue injury) from $48 \%$ to $29 \%$ over a 21 year period, though of course many other factors will be involved.

There is clearly a marked difference in approach between the continental Europeans and the Americans on this issue. There is no hard evidence to support either view, and the approach taken partly depends on logistical factors. As Bircher points out, ${ }^{26}$ German specialist trauma centres are staffed by senior trauma surgeons, able to stabilise the pelvis and deal with abdominal haemorrhage, whereas the need to involve various specialties in the USA or the United Kingdom can make performing aggressive early surgery difficult.

PNEUMATIC ANTISHOCK GARMENT

The modern version of the pneumatic antishock garment (PASG), or MAST suit, came into civilian use after the Vietnam war, and rapidly became standard equipment in prehospital care in the USA. There is some evidence that it can help in the tamponade of pelvic haemorrhage ${ }^{27-29}$ and if fitted in the prehospital phase it can be left on until the external fixator is applied. As it is rarely used in the United Kingdom, ${ }^{30}$ PASG will not be discussed further.

\section{ANGIOGRAPHY AND EMBOLISATION}

The possibility of identifying bleeding vessels angiographically and occluding them with embolic material was recognised in the 1970 s in the USA, and has now been reported from several institutions. Considerable preplanning and organisation are required, in addition to prompt availability of a radiologist experienced in angiography. Experience of this technique in the United Kingdom appears to be limited, with only sporadic use in a few centres.

The first large series published was that by Matalon et al in $1979^{31}-28$ patients, in 20 of whom active bleeding was demonstrated. They reported angiographic control of bleeding in 18 of these 20 , and clinical control in 17 . All patients with pelvic fractures and clinical evidence of associated bleeding were included in the study; angiography was performed within 48 hours of admission, though the authors comment that increasing experience led to earlier angiography as the study progressed. Similarly, Panetta et a ${ }^{21}$ used angiographic embolisation in 31 patients with pelvic fractures who required more than four units of blood in 24 hours, or six in 48 hours. This was a prospective study, but no alternative treatment limbs were used, and complete control of haemorrhage was claimed in $27(87 \%)$, though this was based on repeat arteriography rather than on clinical measurement and outcome. Panetta also notes that the procedure takes on average two and a half hours.

Ben-Menachem et $a l^{10}$ have several times reported favourable results from early embolisation, and have "experienced angiographers and technicians standing by to perform angiography at a moment's notice." Like Burgess et $a l^{29}$ he favours external fixation in 
the resuscitation phase, pointing out that it does not interfere with angiography as long as it is performed rapidly and "not infrequently obviates the need for embolisation."

There are few publications critical of angiographic embolisation and they tend to be rather dated, although there is widespread agreement that the technique will only show a suitable bleeding vessel in 10-15\% of haemodynamically compromised patients. Rothenberger et $a l_{,}^{32}$ for example, found that in only six of 72 pelvic fatalities was there bleeding from a single vessel that they judged to be controllable by operative or angiographic measures.

In these highly selected patients, embolisation seems to be very effective. Mucha ${ }^{17}$ claims those successfully embolised have long term survival rates of up to $80 \%$.

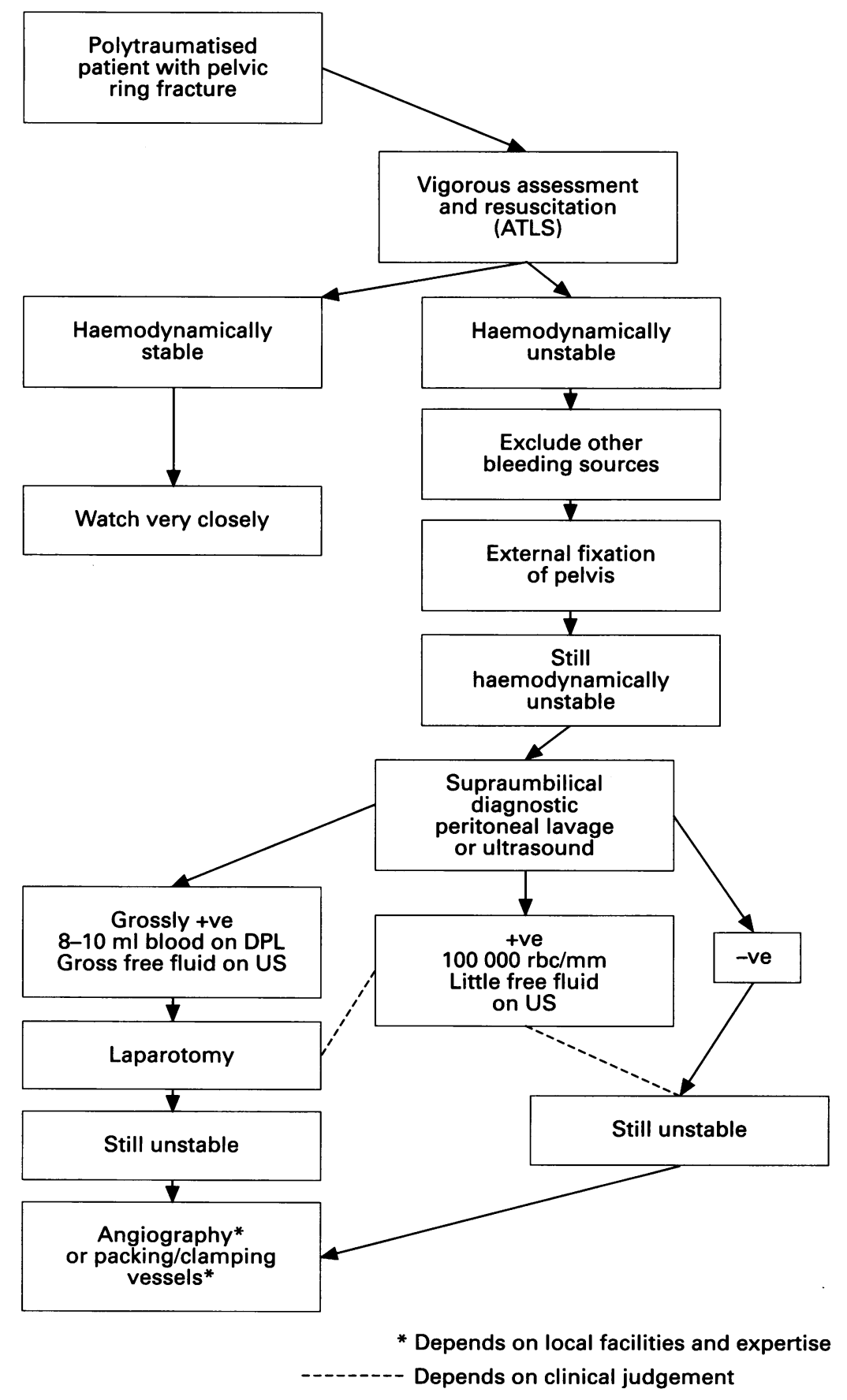

Figure 1 Resuscitation protocol for patient with multiple trauma.

\section{Imaging the abdomen}

Clinical examination is known to be less reliable in multiple trauma, and ATLS (advanced trauma life support) recommends that diagnostic peritoneal lavage is performed to identify occult intraperitoneal injury. In Europe, and increasingly in the United Kingdom, many hospitals use ultrasound for this purpose (Ohiorenoya D, Hilton $M$, personal communication), or computerised tomography in stable patients. The role of diagnostic peritoneal lavage has been questioned in the presence of severe pelvic fractures, because of reported high false positive rates varying from $12.5 \%{ }^{18}$ to $29 \% .^{19}$ There are several possible reasons for this: anterior dissection of blood from the haematoma, diapedesis of red cells across the peritoneum, torn posterior peritoneum encasing the haematoma, or even placement of the trochar into the haematoma. ${ }^{18}$

Hubbard et $a l^{33}$ found a false positive rate of $29 \%$ in patients with pelvic fractures (peritoneal lavage haematocrit $>1 \%$, but negative laparotomy), but this was using an infraumbilical, percutaneous approach. There were no false negative results, a finding roughly in agreement with all studies of diagnostic peritoneal lavage in such patients.

Gilliland et $a l^{18}$ followed diagnostic peritoneal lavage with abdominal angiography in 100 patients, finding a false positive rate of $17 \%$ to $24 \%$. They used a supraumbilical, open method in this retrospective study.

In contrast, Mendez et $a l^{34}$ found a false positive rate of only $0.7 \%$ among 80 positive lavages in patients with pelvic fractures following blunt trauma. In this retrospective study, they report that surgical residents used an open infraumbilical technique, moving to a supraumbilical position if anterior haematoma was encountered. Positive lavage was defined in standard terms. It is claimed that $81 \%$ of diagnostic peritoneal lavages were performed within one hour of injury, and $58 \%$ within 30 minutes-an impressive feat of prehospital and in hospital care. The reason for the very much better results in the Mendez study is probably this short time interval between injury and diagnostic peritoneal lavage, before the haematoma from the pelvis can spread. The relevance to practice in the United Kingdom is doubtful, as achieving such early peritoneal lavage is very difficult in most centres and ultrasound is increasingly preferred (Ohiorenoya $\mathrm{D}$, Hilton $\mathbf{M}$, personal communication).

There is general agreement that a peritoneal lavage that is negative for blood is reliable, and that a grossly positive bloody tap is an indication for immediate laparotomy. Those patients with pelvic fractures, haemodynamic instability, and with a positive lavage only on blood count should have measures directed at controlling presumed pelvic haemorrhage. ${ }^{35}$

Ultrasound has been shown to be at least as effective as diagnostic peritoneal lavage in identifying intra-abdominal injury in patients with blunt trauma patients, ${ }^{36-38}$ and avoids the problem of false positives with pelvic fractures; surgical residents in the USA perform the ultrasound competently, ${ }^{39}$ but frequent use of 
the skill is required to maintain expertise. In the United Kingdom it is more appropriate in all but those few hospitals with high trauma loads for the ultrasound to be performed by radiologists.

\section{Conclusions}

The absence of randomised trials in this field makes firm conclusions difficult, and to some extent management of patients exsanguinating from pelvic fractures will depend on local logistical factors. We believe that "best practice" within the constraints of our current United Kingdom trauma system is as follows: (1) All patients presenting in the United Kingdom with pelvic fractures and haemodynamic instability should be managed in fully staffed accident and emergency departments, preferably by trauma teams led by consultants or experienced middle grade staff, according to ATLS principles. An algorithm is shown in fig 1 (by kind permission of $\mathrm{M}$ Bircher, ${ }^{26}$ with minor changes).

(2) All such patients need to have a diagnostic peritoneal lavage or ultrasound examination of the abdomen to diagnose or exclude intra-abdominal injury. If lavage is grossly positive for blood, or ultrasound positive for free fluid, the abdomen is the likely site of life threatening haemorrhage and immediate laparotomy is indicated, though fitting external fixation before making the skin incision is recommended. If lavage is negative or positive only on blood count, or ultrasound shows little or no fluid, attention should be turned to the pelvis as the source of severe haemorrhage.

(3) External fixation should be applied in the resuscitation phase in all haemodynamically unstable patients with pelvic ring fractures.

(4) In hospitals with the necessary angiographic resources, angiography should be considered in patients who appear to be actively bleeding despite external fixation, correction of clotting abnormalities, and where other sites of bleeding have been excluded. The evidence for benefit in carefully selected patients is strong.

A role in the United Kingdom for surgical control of haemorrhage, with packing and early internal fixation, is unclear. The success reported from Hannover ${ }^{7}$ and other centres is probably partly due to their trauma system as a whole, resulting in a concentration of very traumatised rapidly transported victims: different treatment protocols are needed in this setting and may not be applicable to the United Kingdom unless major changes to our trauma system were to be instituted.

1 Mucha P, Farnell MB. Analysis of pelvic fracture management. J Trauma 1984;24:379-86.

2 Moreno C, Moore EE, Rosenberger A. Haemorrhage associated with major pelvic fractures: a multispecialty challenge. J Trauma 1986;26:987-94.

3 Mendez C, Gubler KD, Maier RV. Diagnostic accuracy of peritoneal lavage in patients with pelvic fractures. Arch Surg 1994;129:477-82.

4 Huittenen VM, Slatis P. Postmortem angiography and dissection of the hypogastric artery in pelvic fractures. Surgery 1973;73:454-62.

5 Latenser BA, Gentilello LM, Tarver AA, Thalgott JS, Batdorf SW. Improved outcome with early fixation of skeletally unstable pelvic fractures. J Trauma 1991;31:28-31.
6 Ganz, R, Krushell RJ, Jakob RP, Kuffer J. The antishock pelvic clamp. Clin Orthop Rel Res 1991;267:71-8.

7 Pohlemann T, Bosch PD, Gansslen A, Tscherne H. The Hannover experience in management of pelvic fractures. Clin Orthop 1994;305:69-80.

8 Schutz M, Stockle U, Hoffmann R, Sudkamp N, Haas N. Clinical experience with two types of pelvic C-clamps for unstable pelvic ring injuries. Injury 1996;27(suppl 1):SA46-50.

9 Tscherne H, Regel G. Care of the polytraumatised patient. J Bone Joint Surg Br 1996;78B:840-52.

10 Ben-Menachem Y, Coldwell DM, Young JWR, Burgess AR. Haemorrhage associated with pelvic fractures: causes,
diagnosis, and emergent management. Am J Radiol 1991;157:1005-14

11 Gylling S, Ward RE, Holcroft JW. Immediate external fixation of unstable pelvic fractures. Am J Surg 1985;150: $721-4$.

12 Riemer BL, Butterfield SL, Diamond DL. Acute mortality associated with injuries to the pelvic ring: the role of early patient mobilisation and external fixation. J Trauma 1993; 35:671-7.

13 McMurtry R, Walton D, Dickinson D, Tile $M$. Pelvic disruption in the polytraumatised patient. Clin Orthop 1980;151:22-30.

14 Latenser BA, Gentilello LM, Tarver AA, Thalgott JS, Batdorf JW. Improved outcome with early fixation of unstable pelvic fractures. J Trauma 1991;31:28-31.

15 Moss MC, Bircher MD. Volume changes within the true pelvis during disruption of the pelvic ring-where does the pelvis during disruption of the pelvic ring-where does

16 Gruen GS, Leit ME, Gruen RE, Peitzman AB. The acute Gruen GS, Leit ME, Gruen RE, Peitzman AB. The acute
management of haemodynamically unstable multiple trauma patients with pelvic ring fractures. J Trauma 1994; 36:706-13.

17 Mucha P, Welch TJ. Haemorrhage in major pelvic fractures. Surg Clin North Am 1988;68:757-73.

18 Gilliland MG, Ward RE, Flynn TC. Peritoneal lavage and angiography in the management of patients with pelvic fractures. Am J Surg 1982;144:744-7.

19 Naam NH, Brown WH, Hurd R. Major pelvic fractures. Arch Surg 1983;118:610-16.

20 Tile M. Fractures of the pelvis and acetabulum. Boston: Williams and Wilkins, 1984 .

21 Panetta T, Sclafani SJA, Goldstein AS. Percutaneous Panetta T, Sclafani SJA, Goldstein AS. Percutaneous
transcatheter embolisation for massive bleeding from pelvic fractures. J Trauma 1985;25:1021-9.

22 Heini PF, Witt J, Ganz R. The pelvic C-clamp for the emergency treatment of unstable pelvic ring injuries. A report on clinical experience of 30 cases. Injury 1996;27(suppl 2):SA38-45.

23 Wilson SC, Cummings DC, Freudigman PT. Prevention of pelvic ring expansion: a biomechanical comparison of fixation methods. Orthop Trans 1993;17:425.

24 Ghanayem AJ, Wilber JH, Lieberman JM, Motta AO. The effect of laparotomy and external fixator stabilisation on pelvic volume in an unstable pelvic injury. J Trauma 1995; pelvic volume

25 Goldstein A, Phillips T, Sclafani SJA, Scalea T, Duncan A, Goldstein J, et al. Early open reduction and internal fixation of the disrupted pelvic ring. J Trauma 1986;26:325-33.

26 Bircher M. Haemorrhage and pelvic fractures. Int J Orthop Trauma 1995;5:76-7.

27 Freeman S. MAST. In: Tintinalli J, Krome RL, Ruiz E, eds. Emergency medicine. A comprehensive study guide. New York: McGraw-Hill, 1988:6-9.

28 Flint LM, Brown A, Richardson JD, Polk HC. Definitive control of bleeding from severe pelvic fractures. Ann Surg 1979;6:709-15.

29 Burgess AR, Eastridge BJ, Young JWR, Ellison TS, Ellison PS, Poka A, et al. Pelvic ring disruptions: effective classification system and treatment protocols. J Trauma classification system

30 Clancy M. Pneumatic antishock garment-does it have a future? J Accid Emerg Med 1995;12:123-5.

31 Matalon TS, Athanasoulis CA, Margolies MN. Haemorrhage with pelvic fractures: efficacy of transcatheter embolisation. Am J Radiol 1979;133:859-64.

32 Rothenberger DA, Fischer RP, Perry JF. Major vascular injuries secondary to pelvic fractures: an unsolved clinical problem. Am J Surg 1978;136:660-2.

33 Hubbard SG, Bivins BA, Sachatello CR, Griffen WO. Diagnostic errors with peritoneal lavage in patients with pelvic fractures. Arch Surg 1979;114:844-6.

34 Mendez C, Gubler KD, Maier RV. Diagnostic accuracy of peritoneal lavage in patients with pelvic fractures. Arch purg 1994;129:477-82.

35 Mucha P. Pelvic fractures. In: Moore EE, et al, eds. Trauma 2nd ed. Norwalk: Appleton and Lange, 1991: 553-69.

36 Liu M, Lee CH, P'eng FK. Prospective comparison of DPL Liu M, Lee CH, P'eng FK. Prospective comparison of DPL,
CT and US for the diagnosis of blunt abdominal trauma. J Trauma 1993;35:267-70.

37 Boulanger BR, Brenneman FD, McLellan BA, Rizoli SB, Culhane J, Hamilton P. A prospective study of emergent abdominal ultrasound after blunt trauma. J Trauma 1995; 39:325-30.

38 McKenny M, Lentz $K$, Nunez D, Soza JL, Sleeman D Axelrad A, et al. Can US replace DPL in the assessment of blunt trauma? J Trauma 1994;37:439-41.

39 Rozyski GS, Ochsner MG, Jaffin JH, Champion HR. Prospective evaluation of surgeons' use of US in the evaluation of trauma patients. J Trauma 1993;34:516-27. 